\title{
Visual persistence at both onset and offset of stimulation
}

\author{
J. T. LINDSAY WILSON \\ University of Stirling, Stirling FK9 4LA, Scotland
}

\begin{abstract}
Subjects identified dot bigrams appearing within or disappearing from random dot patterns. Each letter could be split into two predefined parts; the cues concerning the complete letter were minimized in each half. Identification performance was investigated as the interval between the appearance or disappearance of bigram halves was varied in the range 0 to $300 \mathrm{msec}$. The results indicate that the durations of persistence of information concerning stimulus onset and offset are at least 180 and $120 \mathrm{msec}$, respectively.
\end{abstract}

Visual persistence is conventionally thought of as starting after the end of a stimulus. Coltheart (1977) and Di Lollo (1977) have suggested that persistence begins at or shortly after stimulus onset. Wilson and Phillips (Note 1) have recently proposed that persistence occurs at both onset and offset of stimulation. This paper describes an objective measure which provides evidence that the latter is the case.

One way of attempting to isolate perception of onset and offset is by using stimuli which consist of the appearance or disappearance of elements in complex patterns (e.g., Phillips \& Singer, 1974). Under appropriate conditions, such appearances or disappearances occur almost instantaneously, and their steady state features are masked by the surrounding pattern. Nonetheless, such changes in complex patterns are highly detectable. To the observer, these onsets and offsets appear to last for some limited, though perceptible, time. It is thus possible to estimate the perceptual durations of such events by so-called direct methods. For example, subjects can be asked to press a button as soon as an event begins and, on different trials, as soon as the event appears to have ended. As estimated by the difference between the two sets of reaction times, the phenomenal durations of appearances and disappearances are 240 and $223 \mathrm{msec}$, respectively (Wilson \& Phillips, Note 1 ). This method shares with other direct measures of persistence the problem that one does not know the criteria used by subjects in their judgments. The technique used in the present study is based on an objective measure of persistence developed by Eriksen and Collins (1967, 1968).

Target stimuli were computer-generated dot bigrams; an example of such a bigram is shown at the

The support of a Medical Research Council Training Fellowship is acknowledged. The author would like to thank W. A. Phillips and S. E. Avons for helpful comments and discussion. Please address correspondence to J. T. L. Wilson, Psychology Department, Stirling University, Stirling FK9 4LA, Scotland.

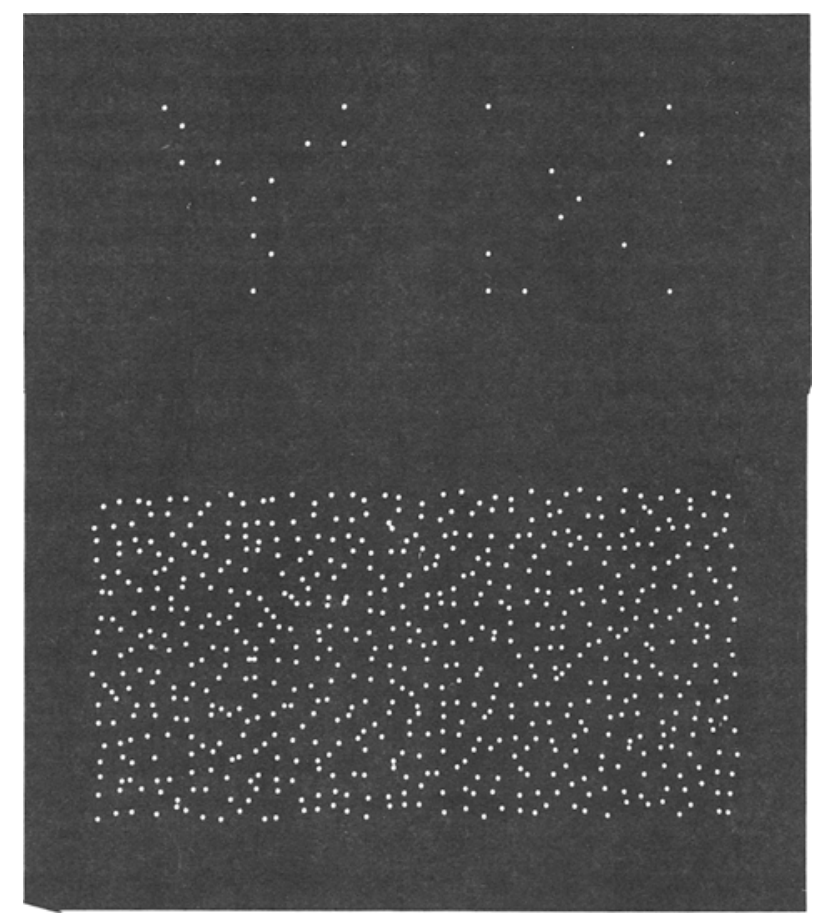

Figure 1. The dot bigram at the top is shown embedded in the random dot pattern at the bottom.

top in Figure 1. These bigrams could be embedded within or subtracted from random-dot patterns. A pattern with an embedded bigram is shown below in Figure 1. As can be seen, the pattern effectively camouflages the letters. It has already been established that single letters appearing or disappearing within such patterns can be accurately identified (Wilson, 1979; Wilson \& Phillips, Note 1). The idea adopted from Eriksen and Collins was of splitting the bigrams into two predefined parts. It was thus possible to investigate bigram recognition as a function of the interval between the appearance or disappearance of the two halves of the target stimulus. 
Two separate experiments were conducted: in one the bigrams were defined by appearances, and in the other, by disappearances. For bigrams defined by appearances, the display sequence was as follows. The random-dot pattern was displayed for $1 \mathrm{sec}$, and then half the target stimulus dots were added. After an interval of between 0 and $300 \mathrm{msec}$, the second half of the target stimulus was added. The random-dot pattern, together with the embedded bigram, was then displayed for a further second. Thus, for this latter period, the entire target stimulus was physically present though camouflaged by surrounding points. Performance of the task requires that information concerning the locations of the dots composing the first half of the target be stored and combined with information concerning the second half. The procedure was similar for the case in which the bigrams were defined by two sets of disappearances.

It is important to stress the main differences between the present paradigm and the Eriksen and Collins paradigm. The stimuli employed by Eriksen and Collins were presented briefly and successively. The bigram halves in the present experiments were not presented successively. The onsets (or offsets) of the bigram halves were successive, but the halves were present together afterwards (or before). Furthermore, the bigram halves used in the present study were displayed for a minimum of $1 \mathrm{sec}$. This figure compares with bigram half durations of $6 \mathrm{msec}$ in Eriksen and Collins' (1967) first experiment. It should be noted that there is evidence that persistence decreases in the Eriksen and Collins paradigm as stimulus duration is increased (Cohene, 1975; Pollack, 1973).

For the sake of convenience, the experiments concerning appearance and disappearance of bigrams are described together in the Method and Results sections.

\section{METHOD}

\section{Subjects}

Each experiment employed 15 psychology students with normal or corrected-to-normal vision. Participation fulfilled a course requirement.

\footnotetext{
Apparatus and Stimuli

Stimuli were presented on a GT40 visual display unit equipped with a P31 phosphor. A chinrest fixed the viewing distance of the screen at $100 \mathrm{~cm}$. At this distance, the screen subtended $12.9 \mathrm{deg}$ horizontally $\times 9.7 \mathrm{deg}$ vertically. Immediately in front of the subject was a response keyboard on which eight consecutive keys were labeled with the eight letters used as stimuli. Ambient illumination was provided by a $12-\mathrm{V}$ dc lamp directed at the GT40 screen; the resulting background luminance of the screen was approximately $5.0 \mathrm{fL}\left(17.2 \mathrm{~cd} / \mathrm{m}^{2}\right)$, as measured with an SEI exposure photometer.

Stimuli were random-dot patterns generated by a host computer. The patterns were constructed by assigning points a random position within each cell of a notional $36 \times 18$ matrix, giving a total of 648 points. The patterns were plotted within an area sub-
}

tending $6.8 \mathrm{deg}$ horizontally $\times 3.4 \mathrm{deg}$ vertically. Each point in the pattern subtended $.06 \mathrm{deg}$ and had a luminance of approximately $12.6 \mathrm{fL}\left(43.2 \mathrm{~cd} / \mathrm{m}^{2}\right)$.

Dot bigrams could be embedded within or subtracted from the random-dot patterns. The letters composing each bigram were chosen by random selection with replacement from a group of eight symmetrical letters of the alphabet: A, H, M, O, U, V, X, Y. Each letter comprised 12 points, including, for some of the letters, extra points which served to make the bigrams more difficult to recognize. Letters could be split into two groups of six points. The points in each half were chosen to minimize cues concerning the resultant letters. At times further cues to the bigrams seemed to be available from the gaps left in the patterns before the target was added or after it was subtracted. This was more pronounced in the latter case. To minimize these cues, some of the dots immediately surrounding the letters were removed from the patterns. In the first experiment, a maximum of 16 points were removed. For the second experiment, this number was increased to 26 and the positions of some of the points in the letters were modified.

The patterns were refreshed every $20 \mathrm{msec}$. During the refresh interval, points were plotted at the maximum rate of the computer: 26 microsec/point. The refresh duration of the entire pattern was $16 \mathrm{msec}$. The time elapsing between plotting the first and last points in a bigram was approximately $13 \mathrm{msec}$.

\section{Procedure}

The subjects were given three blocks of pretraining before the experimental trials. The first block consisted of 20 trials in which subjects identified dot bigrams displayed alone. In the second block, consisting of 50 trials, the subjects identified bigrams which either appeared within (first experiment) or disappeared from (second experiment) random-dot patterns. Finally, the subjects performed the same task with varying intervals between the appearance or disappearance of the two halves of the bigram. Six intervals were used: $0,60,120,180,240$, and $300 \mathrm{msec}$. The subjects performed three trials at each interval, giving a total of 18 trials. The experimental block was similar to the last block of practice except that the subjects performed 20 trials under each condition and were not given knowledge of results. Order of trials was determined by random selection without replacement from the total set of trials for each block.

The task was described to the subjects, and they were given the following printed instructions:

"1. Please fixate the cross before initiating each trial, and attempt to hold your gaze as steady as possible while the pattern is being displayed.

" 2 . There are only eight possible stimulus letters and each of these letters is equally likely to be displayed. The pair of letters displayed can consist of any combination, including the same letter twice. If you are unsure about the letters displayed please guess. Try to avoid thinking for too long-just give your first impression. When responding please give the left letter first then the right letter.

"3. Reaction times are not being recorded in this experiment but please try to complete the task as quickly as possible without making mistakes."

\section{RESULTS}

The subjects varied considerably in their ability to recognize bigrams defined by appearing points. However, all subjects showed a similar effect of interval. The pooled results from the first experiment are shown in Figure 2. The $\mathrm{Y}$ axis in this figure represents the percentage of occasions both letters were identified correctly. Since the letters were each in- 


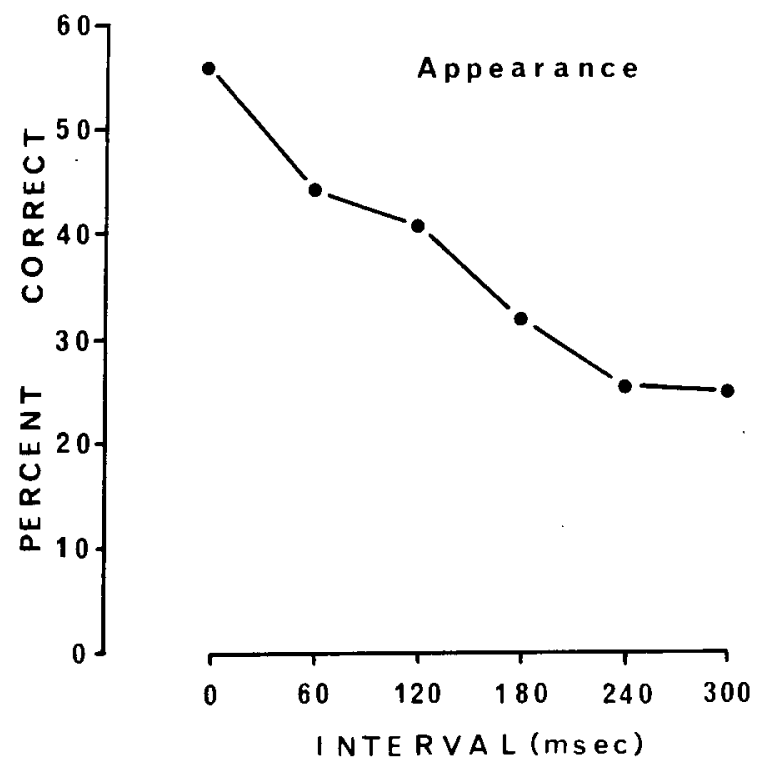

Figure 2. Percentages of correct bigram identifications as a function of the interval between the appearance of the two halves of the bigram.

dependently drawn from a set of eight possibilities, chance performance is $(1 / 8)^{2}$, or $1.6 \%$ correct. A repeated measures analysis of variance was performed on the number of correct responses. The analysis indicated that the effect of interval was highly significant $[F(5,70)=16.2, p<.001]$. As can be seen from Figure 2, performance drops rapidly as the interval between the appearances increases, reaching asymptote between 180 and $240 \mathrm{msec}$. Performance at asymptote is still above chance.

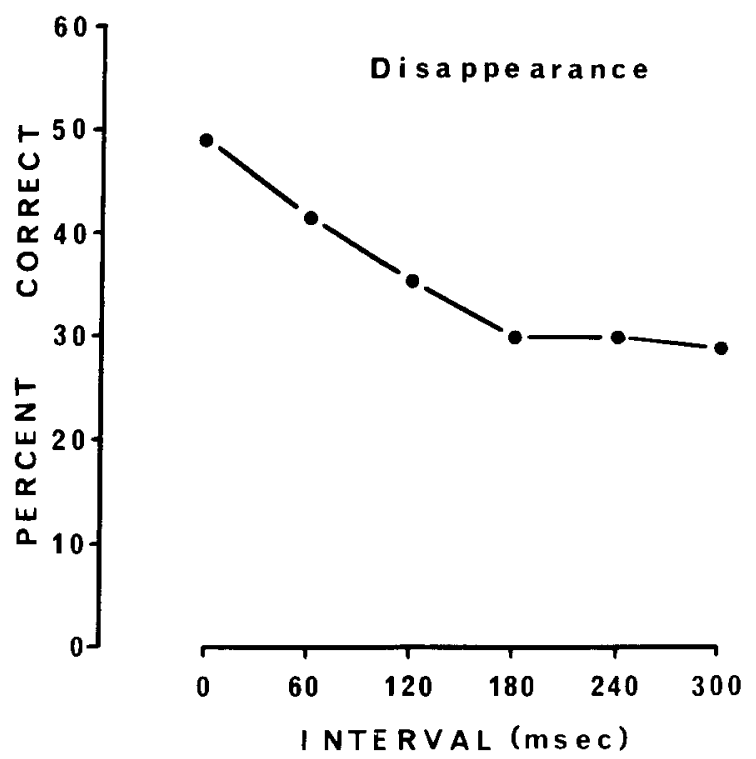

Figure 3. Percentages of correct identifications for bigrams defined by disappearance.
The results for bigrams defined by disappearances are similar. Percentages of correct bigram identifications as a function of the interval between the disappearances of the halves are shown in Figure 3. It should be remembered that the dot stimuli used in this experiment were not identical to those used in the previous experiment: levels of performance in the two experiments are thus not directly comparable. An analysis of variance performed on the number of correct responses indicated that the main effect was highly significant $[F(5,70)=12.1, \mathrm{p}<.001]$. As can be seen from Figure 3, performance falls as the interval increases, apparently reaching asymptote between 120 and $180 \mathrm{msec}$. Performance at asymptote is well above chance.

\section{DISCUSSION}

The results provide evidence for quickly decaying storage at both onset and offset of stimulation. In common with other techniques requiring visual integration, the present method gives an estimate only of the minimum duration of persistence, since a signal may persist for longer than it can be usefully integrated. Under present conditions, these minimum durations of persistence are $180 \mathrm{msec}$ at onset and $120 \mathrm{msec}$ at offset. These findings support those obtained using a direct measure of persistence (Wilson \& Phillips, Note 1).

Some comment should be made concerning the fact that for both appearance and disappearance performance at asymptote is above chance. It was difficult to divide the letters in such a way that the separate halves gave no cues concerning the letter. Part of the performance at asymptote is undoubtedly due to use of cues contained in the separate bigram halves. Furthermore, although the overall stimuli were very complex, the bigrams themselves were not: each bigram half comprised only 12 points. It is well established that there is a short-term visual memory distinct from sensory storage (e.g., Kroll, 1975; Phillips \& Christie, 1977). It seems probable that such a longlasting, low-capacity memory also contributes to performance at asymptote in the present paradigm.

The present findings are consistent with other evidence that there are distinct phases of activity in response to stimulus onset and offset. There is some psychophysical evidence for such "on" and "off" activity (e.g., Boynton, 1972; Holzworth \& Doherty, 1971; Phillips \& Singer, 1974); however, the clearest evidence comes from electrophysiological studies (e.g., Adrian \& Mathews, 1927; Efron, 1964; Hartline, 1938). Varying stimulus parameters such as duration and luminance has well-documented effects on these electrophysiological measures. It might thus prove possible to correlate these effects with the effects the same variables have in the present paradigm.

It is less easy to relate the present results to other 
studies of persistence. Persistence is usually regarded as functioning to maintain the steady state features of the stimulus array. It is clear from the first experiment that this is not its primary function in the present paradigm. The steady state features of bigrams defined by appearances remain present until the offset of the entire dot pattern. The information, which is stored and which decays during the display of the pattern, cannot therefore be information concerning the steady state features of the target stimulus. Rather, what appears to persist is the information that a particular pattern of events has occurred. That is, it is information concerning changes that is stored. The present study thus appears to relate most closely to studies showing persistence of information about movement (Demkiw \& Michaels, 1976; Treisman, Russell, \& Green, 1975).

It seems plausible to suppose that persistence of information concerning changes in the stimulus array may serve as the basis for motion perception. In a series of experiments, Morgan $(1975,1977)$ has shown that differential persistence between the two eyes could account for the Fertsch-Pulfrich illusion of motion in depth. The present study provides further evidence that there is a close link between persistence and event perception. If so, there would be extensive implications for the conception of the function and nature of sensory storage. Further experimentation and a more thorough consideration of the theoretical implications are presented in Wilson (1979).

\section{REFERENCE NOTE}

1. Wilson, J. T. L., \& Phillips, W. A. Event perception as a function for visual sensory storage. Paper presented at the Third European Conference on Visual Perception, Brighton, September 1980.

\section{REFERENCES}

Adrian, E. D., \& Mathews, R. The action of light on the eye. Part I. The discharges of impulses in the optic nerve and its relation to the electric changes in the retina. Journal of Physiology (London), 1927, 63, 378-413.

Boynton, R. M. Discrimination of homogeneous double pulses of light. In D. Jameson \& L. M. Hurvich (Eds.), Handbook of sensory physiology (Vol. 7): Visual psychophysics. Berlin: Springer, 1972.

Cohene, L. S. Iconic memory of dot patterns: Preliminary report. Perceptual and Motor Skills, 1975, 41, 167-170.

Coltheart, M. Contemporary models of the cognitive processes. I: Information input and storage. In V. Hamilton \& M. D. Vernon (Eds.), The development of cognitive processes. London: Academic Press, 1977.

Demkiw, P., \& Michaels, C. Motion information in iconic memory. Acta Psychologica, 1976, 40, 257-264.

Di Lolso, V. Temporal characteristics of iconic memory. Nature, $1977,267,241-243$.

EFron, R. Artificial synthesis of evoked responses to light flash. Annals of the New York Academy of Sciences, 1964, 112 , 292-304.

Eriksen, C. W., \& Collins, J. F. Some temporal characteristics of visual pattern perception. Journal of Experimental Psychology, 1967, 74, 476-484.

Eriksen, C. W., \& Collins, J. F. Sensory traces versus the psychological moment in the temporal organization of form. Journal of Experimental Psychology, 1968, 77, 376-382.

Hartuine, H. K. The response of single optic nerve fibers of the vertebrate eye to illumination of the retina. American Journal of Physiology, 1938, 121, 400-415.

Holzworth, R. J., \& Dohenty, M. E. Visual masking by light offset. Perception \& Psychophysics, 1971, 10, 327-330.

Krolt, N. E. A. Visual short-term memory. In D. Deutsch \& J. A. Deutsch (Eds.), Short-term memory. New York: Academic Press, 1975.

Morgan, M. J. Stereoillusion based on visual persistence. Nature, 1975, 256, 639-640.

Morgan, M. J. Differential visual persistence between the two eyes: A model of the Fertsch-Pulfrich effect. Journal of Experimental Psychology: Human Perception and Performance, 1977, 3. 484-495.

Phillips, W. A., \& Christie, D. F. M. Components of visual memory. Quarterly Journal of Experimental Psychology, 1977, 29, 117-133.

Phillips, W. A., \& Singer, W. Function and interaction of on and off transients in vision. 1. Psychophysics. Experimental Brain Research, 1974, 19, 493-506.

Pollack, I. Interaction effects in successive visual displays: An extension of the Eriksen-Collins paradigm. Perception \& Psychophysics, 1973, 13, 367-373.

Treisman, A., Russell, R., \& Green, J. Brief visual storage of shape and movement. In P. M. A. Rabbitt \& S. Dornic (Eds.), Attention and performance $V$. London: Academic Press, 1975.

WiLson, J. T. L. Event perception and sensory storage. Unpublished doctoral dissertation, University of Stirling, 1979.

(Manuscript received March 25, 1981; revision accepted for publication June 17, 1981.) 